\title{
Existence and continuous dependence for a class of neutral functional differential equations
}

\author{
by LORIS FAINa (Perugia)
}

\begin{abstract}
A general result on existence and continuous dependence of the solution for a quite wide class of N.F.D.E. is given. Further, an abstract equivalence is proved for three different formulations of N.F.D.E.
\end{abstract}

1. Introduction. By a neutral functional differential equation we mean an equation which expresses $\dot{x}(t)$ as a function of present and past values of $x$ and $\dot{x}$. One of the problems linked with such equations is the proper choice of a topological space for solutions. The selection is usually motivated by a desire to give the solutions as much structure as possible, especially continuity of solutions with respect to initial conditions. This problem was investigated by [6, Driver], [11, Hale-Meyer], and [14, 15, Melvin].

It turns out that the space of absolutely continuous functions is a natural choice. The reason is quite simply that the number and location of discontinuities of $\dot{x}$ are not important in determining convergence.

Let $C(\mathbb{R})$ and $L_{1}(\mathbb{R})$ be the spaces of continuous, respectively Lebesgue integrable functions mapping $\mathbb{R}$ into $\mathbb{R}^{n}$ endowed with the compact-open, respectively norm topology.

In the present paper we deal with a Cauchy problem of the following type:

$$
\begin{array}{ll}
\dot{x}(t)=f(t, x, \dot{x}) & \text { a.e. in }\left[t_{0}, t_{0}+p_{0}\right], \\
x(t)=\phi_{0}(t) & \text { in }\left(-\infty, t_{0}\right],
\end{array}
$$

where $f: \mathbb{R} \times C(\mathbb{R}) \times L_{1}(\mathbb{R}) \rightarrow \mathbb{R}^{n}$ satisfies a sort of Carathéodory type conditions and $\phi_{0}:\left(-\infty, t_{0}\right] \rightarrow \mathbb{R}^{n}$ is an absolutely continuous function.

Under very mild conditions, I prove an existence and continuous dependence result for problem (0).

1991 Mathematics Subject Classification: Primary 34A12.

Key words and phrases: neutral functional differential equations, abstract equivalence. 
Similar kinds of problems (0) were considered by [5, Das-Parhi], [13, Kisielewicz], and [15, Melvin]; however, only [5] handled infinite delays, and our assumptions are less restrictive than those of $[5,13,15]$.

Let us observe that the differential equation in (0) contains, as a particular case, a neutral functional differential equation of the form

$$
\dot{x}(t)=f\left(t, x_{t}, \dot{x}_{t}\right) \quad \text { for a.e. } t \in\left[t_{0}, t_{0}+p_{0}\right],
$$

where, for $z:\left(-\infty, t_{0}+p_{0}\right] \rightarrow \mathbb{R}^{n}$, we put $z_{t}(s)=z(t+s)$ for every $t \in\left[t_{0}, t_{0}+p_{0}\right]$ and $s \in(-\infty, 0]$.

In Section 4, we study problem (0) in a much more sophisticated hereditary structure which was introduced in [2, Brandi-Ceppitelli] for studying systems which tend to forget the distant past as it becomes more remote, or systems with sudden memory voids.

I prove here that this general hereditary structure is actually equivalent, in a sense that will be clear in Section 5, to those of problems (0) and (1). This unifying result permits us to study only the most convenient formulation for obtaining results related to the other two. Such an equivalence result was already obtained by [7, Faina] for nonneutral functional differential equations.

Unfortunately, the equation in (0) is not the most general form for a neutral functional differential equation; in fact, in [16, Wang-Wu], [8, Hale], and $[9$, Hale-Cruz], we can find equations of the form

$$
\frac{d}{d t}\left(D\left(t, x_{t}\right)\right)=f\left(t, x_{t}\right)
$$

where $D$ is a continuous operator.

Although the hereditary structure of (2) is less general than that of (0), the presence of a delay in the differentiated term makes the Cauchy problem related to (2) much more difficult than (0).

I am still not able to handle neutral functional differential equations of type (2); I am now working to overcome this difficulty.

2. Notations and statement of the problem. Given a set $E \subset \mathbb{R}$, let $A C(E)$ be the set of all absolutely continuous functions mapping $E$ into $\mathbb{R}^{n}$. Let $\mathcal{W}$ be an open subset of $\mathbb{R} \times C(\mathbb{R}) \times L_{1}(\mathbb{R})$. A pair $\left(t_{0}, \phi_{0}\right) \in$ $\mathbb{R} \times A C\left(\left(-\infty, t_{0}\right]\right)$ is called $\mathcal{W}$-admissible if $\left(t_{0}, \Psi \phi_{0}, 0\right) \in \mathcal{W}\left({ }^{1}\right)$.

$\left({ }^{1}\right)$ Given a function $z:(-\infty, a] \rightarrow \mathbb{R}^{n}$ and $\tau \leq a$, we denote by $\Psi^{\tau} z, \Lambda^{\tau} z$ the functions defined by

$$
\Psi^{\tau} z(t)=\left\{\begin{array}{ll}
z(t) & \text { if } t \in(-\infty, \tau], \\
z(\tau) & \text { if } t \geq \tau,
\end{array} \quad \Lambda^{\tau} z(t)= \begin{cases}z(t) & \text { if } t \in[-\infty, \tau] \\
0 & \text { if } t \geq \tau\end{cases}\right.
$$

For the sake of brevity, we write $\Psi z, \Lambda z$ instead of $\Psi^{a} z, \Lambda^{a} z$. 
Given a function $f: \mathcal{W} \rightarrow \mathbb{R}^{n}$, consider the following Cauchy problem:

$$
\begin{array}{lll}
(\theta .1) & \dot{x}(t)=f(t, x, \dot{x}) & \text { a.e. in }\left[t_{0}, t_{0}+p_{0}\right], \\
(\theta .2) & x(t)=\phi_{0}(t) & \text { in }\left(-\infty, t_{0}\right] .
\end{array}
$$

A solution of problem $(\theta)$ is a function $x \in A C\left(\left(-\infty, t_{0}+p\right]\right)$, with $p \leq p_{0}$, which satisfies equation $(\theta .2)$ and such that $\Psi x$ satisfies equation $(\theta .1)$ in $\left[t_{0}, t_{0}+p\right]$.

We study problem $(\theta)$ for functions $f$ satisfying the following Carathéodory condition $(c)$ :

$\left(c_{1}\right) \quad f(\cdot, x, z)$ is measurable for each fixed $(x, z)$;

$\left(c_{2}\right) \quad f(t, \cdot, \cdot)$ is jointly continuous in $(x, z)$, for almost all $t$, with respect to the compact-open topology in $C(\mathbb{R})$ and the sequential weak convergence in $L_{1}(\mathbb{R})$;

$\left(c_{3}\right) \quad$ for any fixed $\left(t_{0}, x_{0}, z_{0}\right) \in \mathcal{W}$, there is a neighborhood $\mathcal{V}$ of $\left(t_{0}, x_{0}, z_{0}\right)$ and a Lebesgue integrable function $m(\cdot)$ such that $|f(t, x, z)| \leq m(t)$ for every $(t, x, z) \in \mathcal{V}$.

A set of functions $\mathcal{F}$ is said to have property $(c)$ if each function $f \in \mathcal{F}$ has property $(c)$ and $\left(c_{3}\right)$ holds uniformly with respect to $f$.

It is immediately verified that, if $f$ has property $(c)$, then problem $(\theta)$ is equivalent to the following integral equation:

$$
\begin{array}{ll}
x(t)=\phi_{0}\left(t_{0}\right)+\int_{t_{0}}^{t} f(s, x, \dot{x}) d s & \text { in }\left[t_{0}, t_{0}+p_{0}\right], \\
x(t)=\phi_{0}(t) & \text { in }\left(-\infty, t_{0}\right] .
\end{array}
$$

\section{Main results}

3.1. Existence result. Whenever we deal with the space $A C([a, b])$, with $[a, b]$ a finite interval, we consider the norm

$$
\|x\|_{A C([a, b])}=|x(a)|+\int_{a}^{b}|\dot{x}(t)| d t \quad \text { for every } x \in A C([a, b]) .
$$

It is well known that the space $A C([a, b])$, endowed with the topology induced by this norm, is a complete normed space.

Given a subset $Y$ of a metric space $(Z, d)$, we define $B_{Z}(Y ; q)=\{z \in$ $Z: d(z, Y) \leq q\}$.

THEOREM 1. If $f: \mathcal{W} \rightarrow \mathbb{R}^{n}$ has property $(c)$, then for every $\mathcal{U}$-admissible pair $\left(t_{0}, \phi_{0}\right)$ there is at least one solution of the Cauchy problem $(\theta)$.

Proof. By property $\left(c_{3}\right)$, relative to the point $\left(t_{0}, \Psi \phi_{0}, 0\right)$, there is a positive constant not greater than $p_{0}$, which we again denote by $p_{0}$, and a 
Lebesgue integrable function $m(\cdot)$ such that $B_{\mathbb{R} \times C(\mathbb{R}) \times L_{1}(\mathbb{R})}\left(\left(t_{0}, \Psi \phi_{0}, 0\right) ; p_{0}\right)$ $\subset \mathcal{W}$ and

(3) $|f(t, x, z)| \leq m(t)$ for every $(t, x, z) \in B_{\mathbb{R} \times C(\mathbb{R}) \times L_{1}(\mathbb{R})}\left(\left(t_{0}, \Psi \phi_{0}, 0\right) ; p_{0}\right)$.

Let $\delta: \mathbb{R}^{+} \rightarrow \mathbb{R}^{+}$satisfy

(4) $\inf _{\varepsilon>0} \delta(\varepsilon)=0$,

(5) $\quad \int_{I} m(s) d s \leq \varepsilon \quad$ for every interval $I \subset B_{\mathbb{R}}\left(t_{0} ; p_{0}\right)$ with $|I|<\delta(\varepsilon)$.

Let $p>0, p \leq p_{0}$, be such that

$$
\int_{t_{0}}^{t_{0}+p} m(s) d s<p_{0}
$$

Let $H=H\left(t_{0}, \phi_{0}, m(\cdot), p\right)$ be the closed, convex set defined by

$$
\begin{aligned}
H=\left\{y \in A C\left(\left[t_{0}, t_{0}+p\right]\right):\right. & y\left(t_{0}\right)=\phi_{0}\left(t_{0}\right), \\
& |\dot{y}(t)| \leq m(t) \text { a.e. in }\left[t_{0}, t_{0}+p\right], \\
& y([\tau, \tau+\delta(\varepsilon)]) \subset B_{\mathbb{R}^{n}}(y(\tau) ; \varepsilon)
\end{aligned}
$$$$
\text { for every } \left.t_{0} \leq \tau \leq t_{0}+p \text { and } \varepsilon>0\right\} \text {. }
$$

Now, we consider the map $T: H \rightarrow A C\left(\left[t_{0}, t_{0}+p\right]\right)$ defined by

$$
T y(t)=\phi_{0}\left(t_{0}\right)+\int_{t_{0}}^{t} f(s, \widetilde{y}, \dot{\tilde{y}}) d s, \quad t \in\left[t_{0}, t_{0}+p\right],
$$

where

$$
\widetilde{y}(t)= \begin{cases}\phi_{0}(t) & \text { if } t \in\left(-\infty, t_{0}\right] \\ y(t) & \text { if } t \in\left[t_{0}, t_{0}+p\right], \\ y\left(t_{0}+p\right) & \text { if } t \in\left[t_{0}+p, \infty\right) .\end{cases}
$$

By (6), the map $T$ is well defined, that is, $H \subset \mathcal{W}$. By (3)-(5), the range of $T$ is contained in $H$.

We are going to prove that $T$ is a continuous map with relatively compact range. Let $\left(y_{n}\right)_{n \geq 0} \subset H$ with $\left\|y_{n}-y_{0}\right\|_{A C\left(\left[t_{0}, t_{0}+p\right]\right)} \rightarrow 0$ as $n \rightarrow \infty$. From property $\left(c_{2}\right)$, we have

$$
f\left(t, \widetilde{y}_{n}, \dot{\widetilde{y}}_{n}\right) \stackrel{n \rightarrow \infty}{\longrightarrow} f\left(t, \widetilde{y}_{0}, \dot{\widetilde{y}}_{0}\right) \quad \text { for almost all } t \in\left[t_{0}, t_{0}+p\right] .
$$

By (3) and the Lebesgue dominated convergence theorem, $f\left(\cdot, \widetilde{y}_{n}, \dot{\tilde{y}}_{n}\right) \rightarrow$ $f\left(\cdot, \widetilde{y}_{0}, \dot{\widetilde{y}}_{0}\right)$ in $L_{1}\left(\left[t_{0}, t_{0}+p\right]\right)$ as $n \rightarrow \infty$. This shows that $\left(T y_{n}\right)_{n}$ converges to $T y_{0}$ in $A C\left(\left[t_{0}, t_{0}+p\right]\right)$.

Now let $\left(y_{n}\right)_{n} \subset H$. Since the functions $\left(y_{n}\right)_{n}$ are equibounded and equiuniformly continuous, by the Ascoli compactness theorem, there is a subsequence of $\left(y_{n}\right)_{n}$, which we again call $\left(y_{n}\right)_{n}$, and a continuous function 
$y_{0}$ with $y_{n} \rightarrow y_{0}$ in $C\left(\left[t_{0}, t_{0}+p\right]\right)$ as $n \rightarrow \infty$. Since the $\left(\dot{y}_{n}\right)_{n}$ are equiabsolutely integrable, from the Dunford-Pettis compactness theorem, there is a function $w \in L_{1}\left(\left[t_{0}, t_{0}+p\right]\right)$ and a subsequence of $\left(\dot{y}_{n}\right)_{n}$, which we again call $\left(\dot{y}_{n}\right)_{n}$, with $\dot{y}_{n} \rightarrow w$ weakly in $L_{1}\left(\left[t_{0}, t_{0}+p\right]\right)$ as $n \rightarrow \infty$. It is easy to verify that $y_{0} \in A C\left(\left[t_{0}, t_{0}+p\right]\right)$ and $\dot{y}_{0}=w$ a.e. in $\left[t_{0}, t_{0}+p\right]$.

As we proved before, it results that

$$
\left\|T y_{n}-T y_{0}\right\|_{A C\left(\left[t_{0}, t_{0}+p\right]\right)} \stackrel{n \rightarrow \infty}{\longrightarrow} 0 .
$$

As a consequence of the Schauder fixed point theorem, the map $T$ has a fixed point $x$ in $H$. Clearly, the function

$$
\bar{x}(t)= \begin{cases}\phi_{0}(t) & \text { if } t \in\left(-\infty, t_{0}\right], \\ x(t) & \text { if } t \in\left[t_{0}, t_{0}+p\right],\end{cases}
$$

is a solution of problem $(\theta)$.

3.2. A further existence theorem. Let $S$ be a compact subset of $\mathbb{R}$. For every $t \in S$ choose a function $\phi^{t} \in A C((-\infty, t])$.

TheOREM 2. Let $t \rightarrow \Psi \phi^{t}$ be a continuous function from $S$ into $C(\mathbb{R})$, and assume that $Q=\bigcup_{t \in S}\left(t, \Psi \phi^{t}, 0\right)$. Let $\mathcal{F}=\left\{f: \mathcal{W} \rightarrow \mathbb{R}^{n}\right\}$ be a function set with property $(c)$. Then there is a positive number $p$ such that, for every $(t, f) \in S \times \mathcal{F}$, there is a solution $x=x\left(t, \phi^{t}, f\right)$ of $(\theta)$ in $(-\infty, t+p]$.

P r o of. Following Lemma 2 of [4, Ceppitelli-Faina] and Lemma 3 of [2], it is easy to verify that there is a positive number $q$ and a function $m(\cdot) \in$ $L_{1}(\mathbb{R})$ such that $B_{\mathbb{R} \times C(\mathbb{R}) \times L_{1}(\mathbb{R})}(Q ; q) \subset \mathcal{W}$ and

$$
|f(s, x, z)| \leq m(s) \quad \text { for every }(s, x, z) \in B_{\mathbb{R} \times C(\mathbb{R}) \times L_{1}(\mathbb{R})}(Q ; q) \text { and } f \in \mathcal{F} \text {. }
$$

Let $\delta: \mathbb{R}^{+} \rightarrow \mathbb{R}^{+}$be a function with the property (4) and

$$
\int_{I} m(s) d s \leq \varepsilon \quad \text { for every interval } I \subset B_{\mathbb{R}}(S ; q) \text { with }|I|<\delta(\varepsilon) .
$$

Let $p>0, p \leq q$, be such that

$$
\int_{t}^{t+p} m(s) d s<q \quad \text { for every } t \in S .
$$

For fixed $(t, f) \in S \times \mathcal{F}$, let $H_{t}=H\left(t, \phi^{t}, m(\cdot), p\right)$. Similarly to Theorem 1, we prove that the map $T_{t}: H_{t} \rightarrow H_{t}$ given by

$$
T_{t} y(\tau)=\phi^{t}(t)+\int_{t}^{\tau} f(s, \widetilde{y}, \dot{\widetilde{y}}) d s, \quad \tau \in[t, t+p],
$$


where

$$
\widetilde{y}(v)= \begin{cases}\phi^{t}(v) & \text { if } v \in(-\infty, t] \\ y(v) & \text { if } v \in[t, t+p], \\ y(t+p) & \text { if } v \geq t+p\end{cases}
$$

is well defined and satisfies the Schauder theorem's hypotheses. Therefore $T_{t}$ admits a fixed point $x^{t} \in H_{t}$, and the function

$$
\bar{x}^{t}(\tau)= \begin{cases}\phi^{t}(\tau) & \text { if } \tau \in(-\infty, t] \\ x^{t}(\tau) & \text { if } \tau \in[t, t+p],\end{cases}
$$

is a solution of the Cauchy problem $(\theta)$ in $(-\infty, t+p]$.

3.3. Continuous dependence

Theorem 3. Let $\mathcal{F}=\left\{f_{k}: \mathcal{W} \rightarrow \mathbb{R}^{n}: k \in \mathbb{N}\right\}$ be a function set with property $(c)$ and

$$
\lim _{(k, y, w) \rightarrow(+\infty, x, z)} f_{k}(s, y, w)=f_{0}(s, x, z)
$$

for almost all $s$ and for every $(x, z)$. Let $S=\left(t_{k}\right)_{k} \subset \mathbb{R}$ be a sequence converging to $t_{0}$ and $\left(\Psi \phi^{t_{k}}\right)_{k}$ be a sequence converging to $\Psi \phi^{t_{0}}$ in $C(\mathbb{R})$ and in $A C(K)$ for every compact $K \subset \mathbb{R}$. For fixed $p_{0} \in \mathbb{R}^{+}$with $\left[t_{0}, t_{0}+p_{0}\right] \subset$ $\Pi_{\mathbb{R}}(\mathcal{W})\left({ }^{2}\right)$, if the Cauchy problem

$$
\begin{aligned}
& \left(\theta_{k} .1\right) \quad \dot{x}(t)=f_{k}(t, x, \dot{x}) \quad \text { a.e. in }\left[t_{k}, t_{k}+p_{0}\right] \text {, } \\
& \left(\theta_{k} .2\right) \quad x(t)=\phi^{t_{k}}(t) \quad \text { in }\left(-\infty, t_{k}\right] \text {, }
\end{aligned}
$$

has, for $k=0$, a unique solution $x_{0}$ in $\left[t_{0}, t_{0}+p_{0}\right]$, then there is an integer $k_{0}$ such that for $k \geq k_{0}$ there is a solution $x_{k}=x_{k}\left(t_{k}, \phi^{t_{k}}, f_{k}\right)$ of problem $\left(\theta_{k}\right)$, defined in $\left(-\infty, t_{k}+p_{0}\right]$, such that the sequence $\left(\Psi x_{k}\right)_{k}$ converges to $\Psi x_{0}$ in $C(\mathbb{R})$ and in $A C(K)$ for every compact $K \subset \mathbb{R}$.

Proof. Since $x_{0}$ is the unique solution of problem $\left(\theta_{0}\right)$ in $\left[t_{0}, t_{0}+p_{0}\right]$, the compact set $Q=\bigcup_{t \in\left[t_{0}, t_{0}+p_{0}\right]}\left(t, \Psi^{t} x_{0}, 0\right)$ is contained in $\mathcal{W}$.

Following Lemma 2 of [4] and Lemma 3 of [2], it is easy to verify that there is a positive number $q$ and a summable function $m(\cdot)$ such that $B_{\mathbb{R} \times C(\mathbb{R}) \times L_{1}(\mathbb{R})}(Q ; q) \subset \mathcal{W}$ and

$$
|f(s, x, z)| \leq m(s)
$$

for every $(s, x, z) \in B_{\mathbb{R} \times C(\mathbb{R}) \times L_{1}(\mathbb{R})}(Q ; q)$ and for all $f \in \mathcal{F}$. Moreover, since $\left(\Psi \phi^{t_{k}}\right)_{k}$ converges to $\Psi \phi^{t_{0}}$, there is an integer $\bar{k}$ such that

(9) $\quad t_{k} \in B_{\mathbb{R}}\left(t_{0} ; q / 2\right), \quad \Psi \phi^{t_{k}} \in B_{C(\mathbb{R})}\left(\Psi \phi^{t_{0}} ; q / 2\right) \quad$ for every $k \geq \bar{k}$.

$\left({ }^{2}\right)$ Given two topological spaces $Y$ and $Z, \Pi_{Y}: Y \times Z \rightarrow Y$ denotes the standard projection onto $Y$. 
Let $\delta=\delta(\varepsilon): \mathbb{R}^{+} \rightarrow \mathbb{R}^{+}$satisfy

$$
\begin{aligned}
& \inf _{\varepsilon>0} \delta(\varepsilon)=0, \\
& \int_{I} m(s) d s \quad \text { for every interval } I \subset B_{\mathbb{R}}\left(\left[t_{0}, t_{0}+p_{0}\right] ; q\right) \text { with }|I|<\delta(\varepsilon) .
\end{aligned}
$$

Let $p>0, p \leq \inf \left\{p_{0}, q / 2\right\}$, be such that

$$
\int_{t}^{t+p} m(s) d s<q \quad \text { for every } t \in B_{\mathbb{R}}\left(\left[t_{0}, t_{0}+p_{0}\right] ; q / 2\right) .
$$

For fixed $k>\bar{k}$, let $H_{k}=H\left(t_{k}, \phi^{t_{k}}, m(\cdot), p\right)$.

Similarly to Theorem 2, we can prove the existence of a solution $x_{k}^{1}=$ $x_{k}^{1}\left(t_{k}, \phi^{t_{k}}, f_{k}\right) \in H_{k}$ of problem $\left(\theta_{k}\right)$ defined in $\left(-\infty, t_{k}+p\right]$. It is easy to verify that $\left(\Psi x_{k}^{1}\right)_{k}$ is relatively compact in $C(\mathbb{R})$ and $\left(\Lambda \dot{x}_{k}^{1}\right)_{k}$ is locally weakly compact in $L_{1}(\mathbb{R})$. Therefore, there is a function $x \in A C(\mathbb{R})$ such that, upon passing to subsequences, $\Psi x_{k}^{1} \rightarrow x$ in $C(E)$ and $\Lambda \dot{x}_{k}^{1} \rightarrow \dot{x}$ locally weakly in $L_{1}(\mathbb{R})$. By using (8), (7), and $(c)$ we deduce

$$
\begin{array}{lll}
x(\tau)=\phi^{t_{0}}\left(t_{0}\right)+\int_{t_{0}}^{\tau} f_{0}(s, x, \dot{x}) d s, & & \tau \in\left[t_{0}, t_{0}+p\right], \\
x(\tau)=\phi^{t_{0}}(\tau), & & \tau \in\left(-\infty, t_{0}\right],
\end{array}
$$

that is, $x$ is a solution of the Cauchy problem $\left(\theta_{0}\right)$ in $\left[t_{0}, t_{0}+p\right]$.

By the uniqueness for problem $\left(\theta_{0}\right)$, the functions $x$ and $x_{0}$ must coincide in $\left[t_{0}, t_{0}+p\right]$ and, actually, the whole sequence $\left(\Psi x_{k}^{1}\right)_{k}$ converges to $\Psi^{t_{0}+p} x_{0}$ in $C(\mathbb{R})$ and the whole sequence $\left(\Lambda \dot{x}_{k}^{1}\right)_{k}$ converges locally weakly in $L_{1}(\mathbb{R})$ to $\Lambda^{t_{0}+p} \dot{x}_{0}$, i.e. $\left(\Psi x_{k}^{1}\right)_{k}$ converges to $\Psi^{t_{0}+p} x_{0}$ in $A C(K)$ for every compact $K \subset \mathbb{R}$

We proceed by steps of width $p$. There is an integer $\bar{k}_{1}>\bar{k}$ such that, for every $k>\bar{k}_{1}$, we have

$$
t_{k}+p \in\left[t_{0}, t_{0}+2 p\right], \quad \Psi x_{k}^{1} \in B_{C(\mathbb{R})}\left(\Psi^{t_{0}+p} x_{0} ; q / 2\right) .
$$

Let $\bar{S}=\left\{t_{k}+p\right\}_{k \in \mathbb{N}}$; for $k>\bar{k}_{1}$, let $H_{k}^{1}=H\left(t_{k}+p, x_{k}^{1}, m(\cdot), p\right)$. As before, we can prove the existence of a solution $x_{k}^{2} \in H_{k}^{1}$ of problem $\left(\theta_{k}\right)$ defined in $\left(-\infty, t_{k}+2 p\right]$ with initial value $x_{k}^{1}$ at $t_{k}+p$, such that $\left(\Psi x_{k}^{2}\right)_{k}$ converges to $\Psi^{t_{0}+2 p} x_{0}$ in $C(\mathbb{R})$ and in $A C(K)$ for every $K \subset \mathbb{R}$. With a finite number of steps we complete the proof.

4. The general hereditary structure. Let $\mathcal{C}$ and $\mathcal{K}$ be the sets of all closed, respectively compact, nonempty subsets of $\mathbb{R}$.

Let $\alpha: \mathbb{R} \rightarrow \mathcal{C}$ be a multifunction, not necessarily continuous, with $\alpha(t) \subset(-\infty, t]$ for every $t \in \mathbb{R}$. Let $f: \mathcal{W} \rightarrow \mathbb{R}^{n}$ be a function with the Volterra property (with respect to $\alpha$ ): 
$\left(v_{\alpha}\right) \quad$ for every $x_{1}, x_{2} \in C(\mathbb{R}), z_{1}, z_{2} \in L_{1}(\mathbb{R})$, and $t \in \mathbb{R}$, if $\left.x_{1}\right|_{\alpha(t)}=$ $\left.x_{2}\right|_{\alpha(t)},\left.z_{1}\right|_{\alpha(t)}=\left.z_{2}\right|_{\alpha(t)}$ a.e. in $\alpha(t)$, then $f\left(t, x_{1}, z_{1}\right)=f\left(t, x_{2}, z_{2}\right)$.

Given $x \in C(\mathbb{R})$ and $\Omega \in \mathcal{C}$, let $\Gamma(x, \Omega)=\left\{(t, x(t)) \in \mathbb{R}^{n+1}: t \in \Omega\right\}$ be the graph of the restriction of $x$ to $\Omega$ and let $G=\{\Gamma(x, \Omega): x \in C(\mathbb{R})$, $\Omega \in \mathcal{C}\}$ be the set of all graphs. Endow $G$ with a natural topology (see [3, Brandi-Ceppitelli]), and let $\mathcal{X}: G \rightarrow C(\mathbb{R})$ be the continuous function defined as follows (see also [3]): for every $\Omega \in \mathcal{C}$, let $(a, b)$ be the smallest closed interval, bounded or not, containing $\Omega$, and let $\left(a_{i}, b_{i}\right), i \in \mathbb{N}$, be the open intervals whose union is the complement of $\Omega$ in $(a, b)$. For every continuous function $x: \Omega \rightarrow \mathbb{R}^{n}$, we define $\mathcal{X}(\Gamma(x, \Omega))(t)=x(a)$ for $t \in$ $(-\infty, a], \mathcal{X}(\Gamma(x, \Omega))(t)=x(b)$ for $t \in[b, \infty)$ and $\mathcal{X}$ linear in $\left(a_{i}, b_{i}\right), i \in \mathbb{N}$.

Given $p_{0}>0$, put

$$
I_{t_{0}, p_{0}}=\operatorname{cl}\left(\bigcup_{t_{0} \leq t \leq t_{0}+p_{0}}\left(\alpha(t) \cap\left(-\infty, t_{0}\right]\right)\right) \cup\left\{t_{0}\right\} .
$$

A pair $\left(t_{0}, \phi_{0}\right) \in \mathbb{R} \times A C\left(I_{t_{0}, p_{0}}\right)$ is called $\mathcal{W}$-admissible if

$$
\left(t_{0}, \mathcal{X}\left(\Gamma\left(\phi_{0}, I_{t_{0}, p_{0}}\right)\right), 0\right) \in \mathcal{W} .
$$

Consider the following Cauchy problem:

$$
\begin{array}{lll}
\left(\theta_{\alpha} .1\right) & \dot{x}(t)=f(t, x, \dot{x}) & \text { a.e. in }\left[t_{0}, t_{0}+p_{0}\right], \\
\left(\theta_{\alpha} .2\right) & x(t)=\phi_{0}(t) & \text { in } I_{t_{0}, p_{0}} .
\end{array}
$$

A solution of problem $\left(\theta_{\alpha}\right)$ is a function $x \in A C\left(I_{t_{0}, p_{0}} \cup\left[t_{0}, t_{0}+p\right]\right)$, with $0<p \leq p_{0}$, which satisfies equation $\left(\theta_{\alpha} .2\right)$ and such that $\overline{\mathcal{X}} x$ satisfies equation $\left(\theta_{\alpha} .1\right)$ in $\left[t_{0}, t_{0}+p\right]$, where for the sake of brevity, we put

$$
\overline{\mathcal{X}} x(t)= \begin{cases}\mathcal{X}\left(\Gamma\left(\phi_{0}, I_{t_{0}, p_{0}}\right)\right) & \text { if } t \in\left(-\infty, t_{0}\right], \\ \mathcal{X}\left(\Gamma\left(x, I_{t_{0}, p_{0}} \cup\left[t_{0}, t_{0}+p\right]\right)\right) & \text { if } t \in\left[t_{0}, \infty\right) .\end{cases}
$$

The lag function $\alpha$ is not assumed to be continuous nor a compact or connected valued map. Such a lag function was introduced and studied in [2], where its generality was illustrated by examples and references. In [4] and [7], the advantage of studying hereditary differential equations by means of this general lag function was shown.

My aim now is to derive an existence and continuous dependence result for problem $\left(\theta_{\alpha}\right)$ by means of the results proved in the preceding section for the apparently less general problem $(\theta)$.

First of all, we note that if $\left(t_{0}, \phi_{0}\right)$ is a $\mathcal{W}$-admissible pair for problem $\left(\theta_{\alpha}\right)$ then $\left(t_{0}, \mathcal{X}\left(\Gamma\left(\phi_{0}, I_{t_{0}, p_{0}}\right)\right)\right)$ is also $\mathcal{W}$-admissible for problem $(\theta)$.

THEOREM 4 (existence). If $f: \mathcal{W} \rightarrow \mathbb{R}^{n}$ has properties $(c)$ and $\left(v_{\alpha}\right)$, then for every admissible pair $\left(t_{0}, \phi_{0}\right)$ there is at least one solution of the Cauchy problem $\left(\theta_{\alpha}\right)$. 
Pr o of. By Theorem 1, the problem

$\left(\theta_{\mathcal{X}}\right)$

$$
\begin{aligned}
& \dot{x}(t)=f(t, x, \dot{x}) \quad \text { a.e. in }\left[t_{0}, t_{0}+p_{0}\right] \text {, } \\
& x(t)=\mathcal{X}\left(\Gamma\left(\phi_{0}, I_{t_{0}, p_{0}}\right)\right)(t) \quad \text { in }\left(-\infty, t_{0}\right],
\end{aligned}
$$

admits a solution. It is trivial to verify that such a function is also a solution of $\left(\theta_{\alpha}\right)$.

Analogously, we can prove a continuous dependence result for problem $\left(\theta_{\alpha}\right)$, similar to Theorem 3, by using the argument of Theorem 4.

5. An equivalence result. In [7] there is an extensive discussion about the equivalence among some general classes of nonneutral functional differential equations.

I want to adapt that discussion to the classes of neutral functional differential equations introduced in this paper.

Throughout this section, we assume that the data $f$ of problem $(\theta)$ satisfies the Volterra condition $\left(v_{\alpha}\right)$, with $\alpha(t)=(-\infty, t]$ for every $t \in \mathbb{R}$.

Let $\mathcal{H}: \mathbb{R} \times L_{1}(\mathbb{R}) \rightarrow \mathbb{R} \times L_{1}((-\infty, 0])$ be the operator defined by $\mathcal{H}(t, x)=x_{t}$, where $x_{t}(\theta)=x(t+\theta), \theta \in(-\infty, 0]$. Let $U$ be an open subset of $\mathbb{R} \times C((-\infty, 0]) \times L_{1}((-\infty, 0])$ and let $h: U \rightarrow \mathbb{R}^{n}$ be a given function. Put $U_{0}=\Pi_{\mathbb{R}}(U)$. A pair $\left(t_{0}, \phi_{0}\right) \in \mathbb{R} \times A C\left(\left(-\infty, t_{0}\right]\right)$ is called $U$-admissible if $\left(t_{0}, \phi_{0 t_{0}}, 0\right) \in U$. lem:

Given a $U$-admissible pair $\left(t_{0}, \phi_{0}\right)$ we consider the following Cauchy prob-

$$
\begin{array}{lll}
(P .1) & \dot{x}(t)=h\left(t, x_{t}, \dot{x}_{t}\right) & \text { a.e. in }\left[t_{0}, t_{0}+p_{0}\right], \\
(P .2) & x(t)=\phi_{0}(t) & \text { in }\left(-\infty, t_{0}\right] .
\end{array}
$$

A solution of problem $(P)$ is a function $x \in A C\left(\left(-\infty, t_{0}+p\right]\right)$, with $0<p \leq p_{0}$, which satisfies equation $(P .1)$ a.e. in $\left[t_{0}, t_{0}+p\right]$ and equation $(P .2)$ in $\left(-\infty, t_{0}\right]$.

We shall prove here that the three problems $(\theta),\left(\theta_{\alpha}\right)$, and $(P)$ are equivalent. Since the preceding section states the equivalence between problems $(\theta)$ and $\left(\theta_{\alpha}\right)$, we will show only that a suitable problem $(\theta)$, which we denote by $\left(\theta^{*}\right)$, can be associated with every problem $(P)$ so that $\left(\theta^{*}\right)$ and $(P)$ have the same solutions; and vice versa.

For this purpose, given a Cauchy problem $(P)$, consider the continuous function $L: \mathbb{R} \times C(\mathbb{R}) \times L_{1}(\mathbb{R}) \rightarrow \mathbb{R} \times C((-\infty, 0]) \times L_{1}((-\infty, 0])$ defined by

$$
L(t, x, y)=\left(t, x_{t}, y_{t}\right) \text {. }
$$

Note that the open set $\mathcal{W}^{*}=L^{-1}(U)$ is associated with the set $U$ and the function $f^{*}: \mathcal{W}^{*} \rightarrow \mathbb{R}^{n}$ is associated with the function $h$ by $f^{*}(t, x, y)=$ $h\left(t, x_{t}, y_{t}\right)$. 
Furthermore, if $\left(t_{0}, \phi_{0}\right)$ is $U$-admissible then it is also $\mathcal{W}^{*}$-admissible. Therefore the Cauchy problem

$$
\begin{array}{ll}
\dot{x}(t)=f^{*}(t, x, \dot{x}) & \text { a.e. in }\left[t_{0}, t_{0}+p_{0}\right], \\
x(t)=\phi_{0}(t) & \text { in }\left(-\infty, t_{0}\right],
\end{array}
$$

is naturally associated with problem $(P)$.

It is natural to state, for problem $(P)$, similar results to those of Section 3 , which we can prove through problem $\left(\theta^{*}\right)$.

Finally, let us prove that $\left(\theta^{*}\right)$ and $(P)$ have the same solutions. To this end, it is sufficient to observe that, given $x \in A C\left(\left(-\infty, t_{0}+p_{0}\right]\right)$, we have

$$
L(t, \Psi x, \Lambda \dot{x})=\left(t, x_{t}, \dot{x}_{t}\right) .
$$

Now, let problem $(\theta)$ be given and suppose that $\mathcal{W}$ is of the form

$$
\mathcal{W}_{0}=B_{\mathbb{R}}\left(t_{0} ; \beta\right) \times B_{C(\mathbb{R})}\left(\Psi \phi_{0} ; \beta\right) \times B_{L_{1}(\mathbb{R})}(0 ; \beta) .
$$

Consider the continuous function $\xi: \mathbb{R} \times C((-\infty, 0]) \times L_{1}((-\infty, 0]) \rightarrow \mathbb{R} \times$ $C(\mathbb{R}) \times L_{1}(\mathbb{R})$ defined by

$$
\xi(t, x, y)=\left(t, \Psi x_{-t}, \Lambda y_{-t}\right)\left(^{3}\right) .
$$

The open set $U^{*}=\xi^{-1}\left(\mathcal{W}_{0}\right)$ is associated with $\mathcal{W}_{0}$ and the function $h^{*}$ : $U^{*} \rightarrow \mathbb{R}^{n}$ is associated with $f$ by

$$
h^{*}(t, x, y)=f(\xi(t, x, y)) .
$$

Finally, note that since $\left(t_{0}, \phi_{0}\right)$ is $\mathcal{W}_{0}$-admissible then it is also $U^{*}$-admissible. In fact, $\xi\left(t_{0}, \phi_{0 t_{0}}, 0\right)=\left(t_{0}, \Psi \phi_{0}, 0\right) \in \mathcal{W}_{0}$. Thus, the Cauchy problem

$$
\begin{aligned}
& \left(P^{*} .1\right) \quad \dot{x}(t)=h^{*}\left(t, x_{t}, \dot{x}_{t}\right) \quad \text { a.e. in }\left[t_{0}, t_{0}+p_{0}\right] \text {, } \\
& \left(P^{*} .2\right) \quad x(t)=\phi_{0}(t) \quad \text { in }\left(-\infty, t_{0}\right],
\end{aligned}
$$

is naturally associated with problem $(\theta)$.

Let us prove that problems $(\theta)$ and $\left(P^{*}\right)$ have the same solutions. For this purpose, let $x:\left(-\infty, t_{0}+p_{0}\right] \rightarrow \mathbb{R}^{n}$ be a solution of $\left(P^{*}\right)$ on $\left[t_{0}, t_{0}+p_{0}\right]$. We fix a point $\widehat{t} \in\left[t_{0}, t_{0}+p_{0}\right]$ where the equation $\left(P^{*} .1\right)$ is satisfied; this means that

$$
\dot{x}(\widehat{t})=h^{*}\left(\widehat{t}, x_{\hat{t}}, \dot{x}_{\hat{t}}\right) \quad \text { and } \quad \xi\left(\widehat{t}, x_{\hat{t}}, \dot{x}_{\hat{t}}\right)=\left(\widehat{t}, \Psi^{\hat{t}} x, \Lambda^{\hat{t}} \dot{x}\right) \in \mathcal{W}_{0} .
$$

Because of the particular form of $\mathcal{W}_{0}$, we also have

$$
\left(t, \Psi^{\hat{t}} x, \Lambda^{\hat{t}} \dot{x}\right) \in \mathcal{W}_{0} \quad \text { for every } t \in\left[t_{0}, \widehat{t}\right]
$$

$\left({ }^{3}\right)$ Given a function $z:(-\infty, 0] \rightarrow \mathbb{R}^{n}$ and a $t \in \mathbb{R}$, we denote by $z_{-t}$ the function defined by $z_{-t}(\tau)=z(\tau-t)$ for every $\tau \in(-\infty, t]$. 
therefore, in light of property $\left(v_{\alpha}\right)$, the following expression is well defined:

$$
\begin{aligned}
\dot{x}(t) & =h^{*}\left(t, x_{t}, \dot{x}_{t}\right)=f\left(\xi\left(t, x_{t}, \dot{x}_{t}\right)\right) \\
& =f\left(t, \Psi^{t} x, \Lambda^{t} \dot{x}\right)=f\left(t, \Psi^{\widehat{t}} x, \Lambda^{\widehat{t}} \dot{x}\right) \quad \text { a.e. in }\left[t_{0}, \widehat{t}\right] .
\end{aligned}
$$

This proves that $x$ is also a solution of $(\theta)$ in $\left[t_{0}, \widehat{t}\right]$.

Now, let $x$ be a solution of $(\theta)$ in $\left[t_{0}, t_{0}+p_{0}\right]$. Then, for almost all $t \in$ $\left[t_{0}, t_{0}+p_{0}\right]$ we have $(t, \Psi x, \Lambda \dot{x}) \in \mathcal{W}_{0}$. Moreover, observe that $\left(t, \Psi^{t} x, \Lambda^{t} \dot{x}\right) \in$ $\mathcal{W}_{0}$ for almost all $t \in\left[t_{0}, t_{0}+p_{0}\right]$. Therefore,

$$
\begin{aligned}
\dot{x}(t) & =f(t, \Psi x, \Lambda \dot{x})=f\left(t, \Psi^{t} x, \Lambda^{t} \dot{x}\right) \\
& =f\left(\xi\left(t, x_{t}, \dot{x}_{t}\right)\right)=h^{*}\left(t, x_{t}, \dot{x}_{t}\right),
\end{aligned}
$$

and the assertion follows.

\section{References}

[1] R. Bellman and K. Cooke, Differential Difference Equations, Academic Press, New York, 1963.

[2] P. Brandi and R. Ceppitelli, Existence, uniqueness and continuous dependence for hereditary differential equations, J. Differential Equations 81 (1989), 317-339.

[3] -, -, A new graph topology. Connections with the compact-open topology, Appl. Anal., to appear.

[4] R. Ceppitelli and L. Faina, Differential equations with hereditary structure induced by a Volterra type property, submitted for publication.

[5] P. C. Das and N. Parhi, On a functional-differential equation of neutral type, J. Math. Anal. Appl. 35 (1971), 67-82.

[6] R. Driver, Existence and continuous dependence of solutions of a neutral functionaldifferential equation, Arch. Rational Mech. Ann. 19 (1965), 149-166.

[7] L. Fain a, Equivalent hereditary structures for a class of functional differential equations, submitted for publication.

[8] J. K. Hale, Theory of Functional Differential Equations, Appl. Math. Sci. 3, Springer, 1977.

[9] J. K. Hale and M. A. Cruz, Existence, uniqueness, and continuous dependence for hereditary systems, Ann. Mat. Pura Appl. 85 (1970), 63-82.

[10] J. K. Hale and J. Kato, Phase space for retarded equations with infinite delay, Funkc. Ekvac. 21 (1978), 11-41.

[11] J. K. Hale and K. R. Meyer, A class of functional equations of neutral type, Mem. Amer. Math. Soc. 76 (1967).

[12] D. Henry, Linear autonomous neutral functional differential equations, J. Differential Equations 15 (1974), 106-128.

[13] M. Kisielewicz, Some generic properties of functional-differential equations of neutral type, J. Math. Anal. Appl. 97 (1984), 229-244.

[14] W. R. Melvin, Topologies for nonlinear functional differential equations, J. Differential Equations 13 (1973), 24-31. 
[15] W. R. Melvin, A class of neutral functional-differential equations, ibid. 12 (1972), 524-534.

[16] Z. Wang and $\mathrm{J} . \mathrm{Wu}$, Neutral functional differential equations with infinite delay, Funkc. Ekvac. 28 (1985), 157-170.

Dipartimento di Matematica

Via L. Vanvitelli 1

06100 Perugia, Italy

Reçu par la Rédaction le 16.6.1993 